\title{
Quality Improvement on MRI Corrupted with Rician Noise using Wave Atom Transform
}

\author{
Virender Kumar \\ Department of ECE \\ SJPML, Radaur, India
}

\author{
Sonia Saini \\ Department of ECE \\ SJPML, Radaur, India
}

\author{
Sanjeev Dhiman \\ Department of ECE \\ BMSCE, Muktsar, India
}

\begin{abstract}
Magnetic resonance imaging is a medical imaging technique that measures the response of atomic nuclei of body tissues to high frequency radio waves when placed in a strong magnetic field and that produces images of the internal organs. Denoising is always a challenging problem in magnetic resonance imaging and important for clinical diagnosis and computerized analysis, such as tissue classification and segmentation. It is well known that the noise in magnetic resonance imaging has a Rician distribution. . In this paper, an improved de-noising technique is proposed on Magnetic Resonance Images highly corrupted with Rician Noise using wave atom shrinkage.
\end{abstract}

\section{General Terms}

Clinical diagnosis, tissue classification, segmentation.

\section{Keywords}

De-noising, Histogram, Magnetic Resonance Image, Rician Noise, Variance Estimation, Wave Atom Transform.

\section{INTRODUCTION}

Wave atoms are a recent addition to the repertoire of mathematical transforms of computational harmonic analysis. They come either as an orthonormal basis or a tight frame of directional wave packets, and are particularly well suited for representing oscillatory patterns in images. They also provide a sparse representation of wave equations, hence the name wave atoms [1]. Magnetic Resonance Imaging (MRI) is a notable medical imaging technique that has proven to be particularly valuable for examination of the soft tissues in the body. MRI is primarily used to demonstrate pathological or other physiological alterations of living tissues and is a commonly used form of medical imaging. Because of the resolution of MRI and the technology being essentially harmless it has emerged as the most accurate and desirable imaging technology [2]. Despite significant improvements in recent years, magnetic resonance (MR) images often suffer from low Signal to Noise Ratio (SNR) especially in brain imaging. This paper presents an improved multi resolution denoising method to de- noise Magnetic Resonance Images using Wave Atom Shrinkage, histogram based noise variance estimation [3] and modified threshold calculation that leads to the improvement of SNR in high noise level images. The paper is organized with sections as follows. In section 2, the work related to this paper is briefly explained, Section 3 briefly explained about the rician noise which is usually present in MRI, Section 4 deals with the explanation of the estimation of rician noise variance, used in this method, In section 5, the theoretical concepts of wave atom transforms is described, in section 6, the application of wave atom transform and wavelet transforms to MRI and observations are discussed. In section 7, the paper is concluded by briefly explained the pros and corns of the proposed method.

\section{RELATED WORKS}

The image processing literature presents a variety of denoising methods. Many of the popular de-noising algorithms suggested are based on wavelet thresholding [4]-[7]. These approaches attempt to separate significant features from noise in the frequency domain and simultaneously preserve them while removing noise. If the wavelet transform is applied on MR magnitude data directly, both the wavelet and the scaling coefficients of a noisy MRI image become biased estimates of their noisefree counterparts. Therefore, it was suggested [5] that the application of the wavelet transform on squared MR magnitude image data (which is noncentral chi-square distributed) would result in the wavelet coefficients no longer being biased estimates of their noise-free counterparts. Although the bias still remains in the scaling coefficients, it is not signal-dependent and can therefore be easily removed [5], [7]. The difficulty with wavelet or anisotropic diffusion algorithms is again the risk of over-smoothing fine details particularly in low SNR images [8]. From these points, it is understood that all the algorithms have the drawback of over-smoothing fine details. In [9], stated that oscillatory functions or oriented textures have a significantly sparser expansion in wave atoms than in other fixed standard representations like Gabor filters, wavelets and curvelets. In [10], denoising using Wave Atom is done by estimating the noise variance by trial and error method. In [11], denoising using Wave Atom is done by estimating the noise variance by histogram technique.

\section{RICIAN NOISE}

The image intensity in magnetic resonance magnitude images in the presence of noise is to be governed by a Rician distribution. Rician noise depends on the data itself, it is not additive, so to add Rician noise to data, what we really mean is make the data Rician distributed [12],[13]. The magnetic resonance signals are acquired in quadrature channels. Each signal produces an image that is degraded by a zeromean Gaussian noise of standard deviation $\sigma_{0}$ (which we define as the noise level). The two images are then combined into a magnitude image and the Gaussian noise PDF is transformed into a Rician noise PDF. The joint probability density of the noise from two quadrature channels can be expressed as [14]:

$$
p\left(n_{r}, n_{i}\right)=\frac{1}{2 \pi \sigma_{0}^{2}} \exp \left(-\frac{n_{r}^{2}+n_{i}^{2}}{2 \pi \sigma_{0}^{2}}\right)
$$

The expectation values for the mean magnitude and the variance are [12]: 


$$
\begin{aligned}
& \mathrm{I}=\sigma_{0} \sqrt{\frac{\pi}{2}} \exp \left(-\frac{X^{2}}{4 \sigma_{0}^{2}}\right) \times \\
& {\left[\left(1+\frac{X^{2}}{2 \sigma_{0}^{2}}\right) I_{0}\left(\frac{X^{2}}{4 \sigma_{0}^{2}}\right)+\frac{X^{2}}{2 \sigma_{0}^{2}} I_{1}\left(\frac{X^{2}}{4 \sigma_{0}^{2}}\right)\right]} \\
& \sigma_{I}^{2}=X^{2}+2 \sigma_{0}^{2}-\frac{\pi \sigma_{0}^{2}}{2} \exp \left(\frac{X^{2}}{2 \sigma_{0}^{2}}\right) \times \\
& {\left[\left(1+\frac{X^{2}}{2 \sigma_{0}^{2}}\right) I_{0}\left(\frac{X^{2}}{4 \sigma_{0}^{2}}\right)+\frac{X^{2}}{2 \sigma_{0}^{2}} I_{1}\left(\frac{X^{2}}{4 \sigma_{0}^{2}}\right)\right]^{2}}
\end{aligned}
$$

where $I_{0}$ and $I_{1}$ are modified Bessel functions of the first kind and $\mathrm{X}$ denote the MR magnitude image.

\section{NOISE VARIANCE ESTIMATION}

Noise variance estimation plays an important role for the proper selection of threshold in the multi-resolution techniques. Many noise variance estimation methods are available in the literature; here the Automatic estimation of the noise variance from the histogram of an MR image developed in [3] is used. This method is superior in terms of the mean squared error. Let $\left\{l_{i}\right\}$ with $\mathrm{i}=0, \ldots, \mathrm{K}$ denote the set of boundaries of histogram bins. Furthermore, let $n_{i}$ represent the number of observations (counts) within the bin $\left[l_{i-1}, l_{i}\right]$, which are multinomially distributed. Then, the joint PDF of the histogram data is given by :

$$
p\left(\left\{n_{i}\right\} / \sigma,\left\{l_{i}\right\}\right)=\frac{N_{K} !}{\prod_{i=1}^{K} n_{i} !} \prod_{i=1}^{K}(\sigma)
$$

with $N_{K}=\sum_{i=1}^{K} n_{i}$ the total number of observations within the partial histogram and $p_{i}$ the probability that an observations within the partial the range $\left[l_{i-1}, l_{i}\right]$. For Rayleigh distributed observations, this probability is given by

$$
p_{i}(\sigma)=\frac{\int_{l_{i-1}}^{l_{i}} \frac{m}{\sigma^{2}} \exp \left(-\frac{m^{2}}{2 \sigma^{2}}\right) d m}{\sum_{i=1}^{K} \int_{l_{i-1}}^{l_{i}} \frac{m}{\sigma^{2}} \exp \left(-\frac{m^{2}}{2 \sigma^{2}}\right) d m}
$$

Since

$$
\int_{a}^{b} \frac{m}{\sigma^{2}} \exp \left(-\frac{m^{2}}{2 \sigma^{2}}\right) d m=e^{-\frac{a^{2}}{2 \sigma^{2}}}-e^{-\frac{b^{2}}{2 \sigma^{2}}}
$$

Using (6), (5) simplifies to

$$
p_{i}(\sigma)=\left(e^{-\frac{t_{i-1}^{2}}{2 \sigma^{2}}}-e^{-\frac{t_{i}^{2}}{2 \sigma^{2}}}\right)\left(e^{-\frac{t_{0}^{2}}{2 \sigma^{2}}}-e^{-\frac{t_{K}^{2}}{2 \sigma^{2}}}\right)^{-1}
$$

If the set of observations $\left\{n_{i}\right\}$ is fixed and $\sigma$ is regarded as a variable, the joint PDF given in (4) is called a likelihood function. The ML estimate is then found by maximizing this likelihood function $\mathrm{L}$ with respect to $\sigma$ :

$$
\bar{\sigma}_{M L, K}=\arg \max L\left(\sigma /\left\{n_{i}\right\},\left\{l_{i}\right\}\right)
$$

Equivalently, the ML estimate of $\sigma$ is found by minimizing $-\ln \mathrm{L}$ with respect to $\sigma$ :

$$
\begin{aligned}
\hat{\sigma}_{M L, K=\arg \max } & {\left[N_{K} \ln \left(e^{-\frac{t_{0}^{2}}{2 \sigma^{2}}}-e^{-\frac{t_{K}^{2}}{2 \sigma^{2}}}\right)\right.} \\
& \left.-\sum_{i=1}^{K} n_{i} \ln \left(e^{-\frac{t_{i-1}^{2}}{2 \sigma^{2}}}-e^{-\frac{t_{i}^{2}}{2 \sigma^{2}}}\right)\right]
\end{aligned}
$$

Eq. (9) is the ML estimate of the noise standard deviation from $\mathrm{K}$ bins. This result can be interpreted as follows. The joint PDF (4) with the ML estimate (9) as parameter generates the set of observations (counts) from which this parameter is estimated with a larger probability than a joint PDF with any other value of $\sigma$. For implementation software, we refer to the homepage http://visielab.ua.ac.be/staff/poot/BackgroundNoiseLvlEst.z.

\section{WAVE ATOM}

\subsection{Generalization}

A complete collection of wave packets $\varphi_{\mu}(x)$ must "span" all positions and frequencies; we will call it a phase-space tiling, with wave packets as tiles. Universality it is suggested that two parameters should suffice to index a lot of known wave packet architectures: $\alpha$ to index whether the decomposition is "multiscale" $(\alpha=1)$ or not $(\alpha=0)$; and $\beta$ to indicate whether basis elements should be isotropic $(\beta=\alpha)$ or on the contrary, elongated and anisotropic $(\beta<\alpha)$. In terms of phase-space localization of the wave packets, we will require that

- the essential support of $\varphi_{\mu}(x)$ be of size $\sim 2^{-\alpha j}$ vs. $2^{-\beta j}$ as scale $j$, with oscillations of wavelength $\sim 2^{-j}$ tranverse to the ridge; and

- $\quad$ the essential support of $\hat{\varphi}_{\mu}(\xi)$ be size $\sim 2^{\alpha \mathrm{j}}$ vs. $2^{\beta \mathrm{j}}$ as scale $\mathrm{j}$, at a distance $\sim 2^{\mathrm{j}}$ from the origin.

In terms of $\alpha$ and $\beta$, we will clarify the connections between various transforms. Curvelets corresponds to $\alpha=1, \beta=1 / 2$, wavelets are $\alpha=\beta=1$, ridgelets are $\alpha=1, \beta=0$, and the Gabor transforms is $\alpha=\beta=0$. This situation is summarized in figure 1 . Wave Atoms corresponds to $\alpha=\beta=1 / 2$, having an aspect ratio 
$\sim 2^{-j / 2} \times 2^{-j / 2}$ in space, with oscillations of wavelength $\sim 2^{-j}$ in the codirection $\xi_{\mu}$. Wave atoms come from property that they also provide an optimally sparse representation of wave propagators with applications to fast numerical solvers for wave equations.

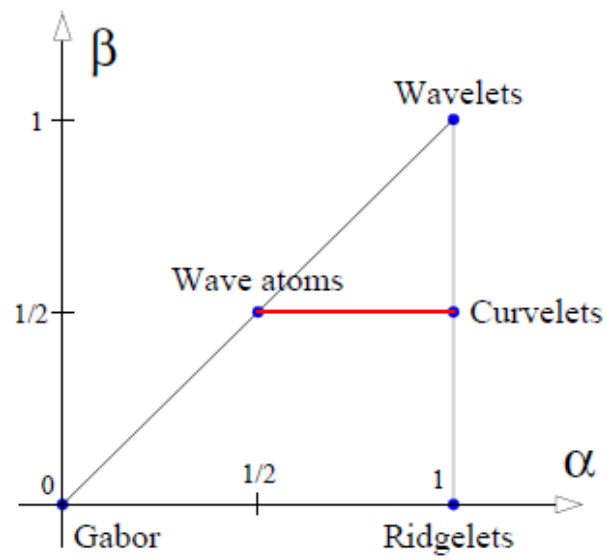

Fig. 1: Identification of various transforms as $(\alpha, \beta)$ families of wave packets. The horizontal segment at $\beta=1 / 2$ indicates the only wave packet families that yield sparse decompositions of Fourier Integral Operators.

\subsection{Definition of Wave Atoms}

We write wave atoms as $\varphi_{\mu}(x)$, with subscript $\mu=(\mathrm{j}, \mathrm{m}, \mathrm{n})=\left(\mathrm{j}, \mathrm{m}_{1}, \mathrm{~m}_{2}, \mathrm{n}_{1}, \mathrm{n}_{2}\right)$. All five quantities $\mathrm{j}, \mathrm{m}_{1}, \mathrm{~m}_{2}, \mathrm{n}_{1}, \mathrm{n}_{2}$ are integer-valued and index a point $\left(\mathrm{x}_{\mu}, \xi_{\mu}\right)$ in phase-space, as $x_{\mu}=2^{-j} n, \xi_{\mu}=\pi 2^{j} m, C_{1} 2^{j} \leq \max _{i=1,2}\left|m_{i}\right| \leq C_{2} 2^{j}$

Where $\mathrm{C}_{1}, \mathrm{C}_{2}$ are two positive constants whose values are implied by the specifics of the implementation. The position vector $x_{\mu}$ is the center of $\varphi_{\mu}(x)$ and the wave vector $\xi_{\mu}$ determines the centers of both bumps of $\hat{\varphi}_{\mu}(\xi)$ as $\pm \xi_{\mu}$. Wave atoms as a variant of 2-D wavelet packets and obey the parabolic scaling of curvelets wavelength $=(\text { diameter })^{2}$. Oscillatory functions or oriented textures (e.g., fingerprint, seismic profile, engineering surfaces) have a significantly sparser expansion in wave atoms than in other fixed standard representations like Gabor filters, Wavelets, and Curvelets. Wave atoms have the ability to adapt to arbitrary local directions of a pattern, and to sparsely represent anisotropic patterns aligned with the axes.

In the following, we shortly summarize the wave atom transform as recently suggested in [2]. More detail can be found in [14]. Consider a 1-D family of wave packets $\psi_{m, n}^{j}(x), \mathrm{j} \geq 0, \mathrm{~m} \geq 0, n \in N$, centered in frequency around $\pm w_{j, m}= \pm \pi 2^{j} m$ with $c_{1} 2^{j} \leq m \leq c_{2} 2^{j}$ (where $\mathrm{c}_{1}<\mathrm{c}_{2}$ are positive constants) and centered in space around $x_{j, n}=2^{-j} n$. For that purpose, let $\mathrm{g}$ be a real valued $C^{\infty}$ bumb function with compact support in $[-7 \pi / 6,5 \pi / 6]$ such that for $|\omega| \leq \pi / 3$

$$
g\left(\frac{\pi}{2}-\omega\right)^{2}+g\left(\frac{\pi}{2}+\omega\right)=1
$$

$$
g\left(-\frac{\pi}{2}-2 \omega\right)^{2}=g\left(\frac{\pi}{2}+\omega\right)
$$

Then the function $\hat{\psi}_{m}^{0}(\omega)$ is determined by the formula

$$
\begin{aligned}
e^{-\omega / 2}\left[e^{i \omega n} g\left(e_{m}\left(\omega-\pi\left(m+\frac{1}{2}\right)\right)\right)\right. \\
\left.+e^{-i \omega n} g\left(e_{m+1}\left(\omega+\pi\left(m+\frac{1}{2}\right)\right)\right)\right]
\end{aligned}
$$

Where $e_{m}=(-1)^{m}$ and $\alpha_{m}=(\pi / 2)(m+1 / 2)$. The properties of $g$ have to ensure that

$$
\sum_{m=0}^{\infty}\left|\hat{\psi}_{m}^{0}(\omega)\right|^{2}=1
$$

Then translates $\left\{\psi_{m}^{0}(.-n)\right\}$ form an orthonormal basis $L^{2}(R)$. Introducing the basis functions

$\psi_{m, n}^{j}(x)=\psi_{m}^{j}\left(x-2^{-j} n\right)=2^{j / 2} \psi_{m}^{0}\left(2^{j} x-n\right)$

The transform $W A: L^{2}(R) \rightarrow l^{2}(Z)$ maps a function u onto a sequence of wave atom coefficients

$$
c_{j, m, n}=\int_{-\infty}^{\infty} u(x) \psi_{m, n}^{j}(x) d x=\frac{\frac{1}{2 \pi} \int_{-\infty}^{\infty}\left[\left(e^{-i 2^{-j}} n \omega\right) \times\right.}{\left.\psi_{m}^{j}(\omega) \hat{\mu}(\omega) d \omega\right]}
$$

In the 2-D case, Let $\mu=(j, m, n)$. Where $m=\left(m_{1}, m_{2}\right)$ and $\mathrm{n}=\left(\mathrm{n}_{1}, \mathrm{n}_{2}\right)$. We consider

$$
\phi_{\mu}^{+}\left(x_{1}, x_{2}\right)=\psi_{m_{1}, n_{1}}^{j}\left(x_{1}\right) \psi_{m_{2}, n_{2}}^{j}\left(x_{2}\right)
$$

and, the Hilbert transform wavelet packets

$$
\phi_{\mu}^{-}\left(x_{1}, x_{2}\right)=\psi_{m_{1}, n_{1}}^{j}\left(x_{1}\right) \psi_{m_{2}, n_{2}}^{j}\left(x_{2}\right)
$$

Where for decomposition

$\psi_{m, n}(\omega)=\psi_{m, n,+}^{j}(\omega)+i \psi_{m, n,-}^{j}(\omega)$ with

$\psi_{m, n,+}^{j}(\omega)=\psi_{m, n,-}^{j}(\omega)$ and the Hilbert transform is defined by

$$
\hat{H} \psi_{m, n}^{j}(\omega)=-i \psi_{m, n,+}^{j}(\omega)+i \psi_{m, n,-}^{j}(\omega)
$$

(Note that the above decomposition of $\hat{\psi}_{m, n}$ is possible since $\psi_{m, n}$ is real valued). A recombination

$$
\psi_{\mu}^{(1)}=\frac{\psi_{\mu}^{+}+\psi_{\bar{\mu}}}{2}, \psi_{\mu}^{(2)}=\frac{\psi_{\bar{\mu}}+\psi_{\bar{\mu}}}{2}
$$

Provides basis functions with two bumps in the frequency plane being symmetric with respect to the origin. Together, $\psi_{\mu}^{(1)}$ and $\psi_{\mu}^{(2)}$ form a wave atom frame, and the wave atom coefficients $C_{u}^{(1)}, C_{u}^{(2)}$ are the scalar products of u with 
$\psi_{\mu}^{(1)}$ and $\psi_{\mu}^{(2)}$.In [2], a discretization of this transform is described for the 1-D case, as well as an extension to two dimensions. The algorithm is based on the fast Fourier transform and a wrapping trick. For implementation software, we refer to the homepage http://www.waveatom.org/ software.html due to Demanet and Ying.

\section{EXPERIMENTS AND RESULTS}

This section gives a detailed study of the proposed MRI denoising algorithm. It compares the performance of the proposed method with wavelet shrinkage. It also compares performance of new proposed threshold with old threshold. It is evaluated with simulated images and real images.

\subsection{Simulated Images}

For experiments with simulated images, images were loaded from Matlab 7.8.0(R2009a) software. Matlab includes an MRI data set that contains 27 image slices of a human head. Results are verified for all 27 image slices. The matlab code to generate rician noise is down loaded from [15]. Noisy image with different noise levels are applied on the proposed denoising method and the Peak Signal to Noise ratio (PSNR) is obtained by

$$
p s n r=10 \log 10\left(\frac{R^{2}}{M S E}\right)
$$

Here $R$ is the maximum fluctuation in the input image data type. For example, if the input image has a double-precision floating-point data type, then $R$ is 1 . If it has an 8-bit unsigned integer data type, $R$ is 255 , etc. We used double-precision precision floating-point data type. MSE (mean square error) is given as

$$
m s e=\frac{1}{m^{*} n} \sum_{i=1}^{m} \sum_{j=1}^{n}(N(i, j)-D N(i, j))^{2}
$$

Where $m$ is number of rows in the image, $N(i, j)$ is the noisy image and $\mathrm{DN}(\mathrm{i}, \mathrm{j})$ is the de-noised image. Signal to Noise ratio (SNR) is obtained by

$$
s n r=10 \log 10\left(\frac{\operatorname{var}(x)}{\operatorname{var}(\hat{x}-x)}\right)
$$

Where $\mathrm{x}$ is noise free simulated images and $\hat{x}$ is the noisy image or de-noised images.

\subsection{Real Images}

The real images were down loaded from the Open Acess Series of imaging Studies (OASIS) database [16]. Results are verified for these images. Old threshold given in [11] is given as:

$$
\text { Old threshold }=\sqrt{\ln (\max v a l-\min v a l)} \sigma
$$

Modified new threshold is given as:

$$
\text { New threshold }=\sqrt{\ln \left((\max v a l)^{2}-(\min v a l)^{2}\right)} \sigma
$$

Where $\sigma$ the noise variance, maxval is the highest pixel value in the image and the minval is the lowest pixel value in the image. We obtained better results in case of modified

threshold for real and simulated images. Noise variance is estimated by the method given in [3].

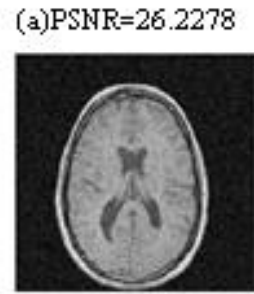

(d)PSHR=32.8246
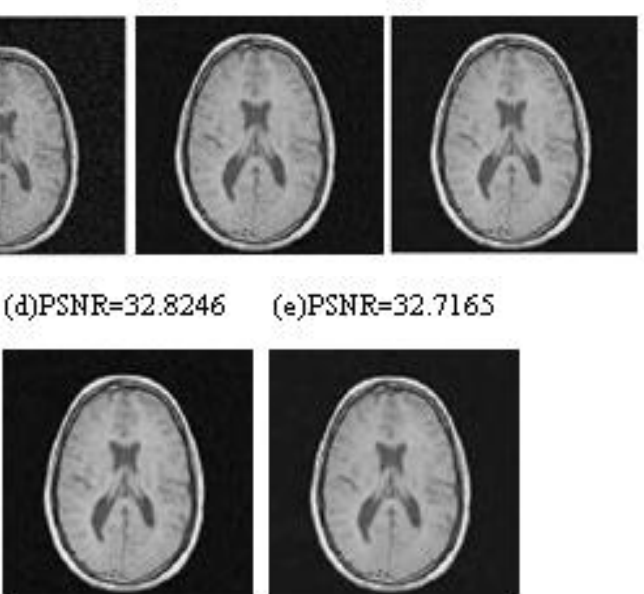

(e)PSHR $=32.7165$

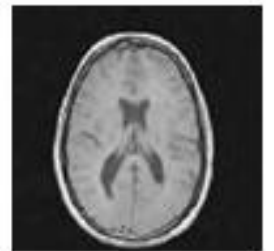

Fig 1: High SNR Images

(a)Noisy image

(b)De-noised using Wave Atom with old threshold

(c) De-noised using Wavelet with old threshold

(d) De-noised using Wave Atom with new threshold

(e) De-noised using Wavelet with new threshold

Different quality parameters for above denoised images are given in table 1 .

Table 1. Quality Parameters for High SNR Images

\begin{tabular}{|l|l|l|l|l|}
\hline $\begin{array}{l}\text { Quality } \\
\text { parameters }\end{array}$ & $\begin{array}{l}\text { Denoised } \\
\text { using } \\
\text { Wave } \\
\text { Atom } \\
\text { with old } \\
\text { threshold }\end{array}$ & $\begin{array}{l}\text { Denoised } \\
\text { using } \\
\text { Wavelet } \\
\text { with old } \\
\text { threshold }\end{array}$ & $\begin{array}{l}\text { Denoised } \\
\text { using } \\
\text { Wave } \\
\text { Atom } \\
\text { with new } \\
\text { threshold }\end{array}$ & $\begin{array}{l}\text { Denoised } \\
\text { using } \\
\text { Wavelet } \\
\text { with new } \\
\text { threshold }\end{array}$ \\
\hline $\begin{array}{l}\text { MSE(mean } \\
\text { square error) }\end{array}$ & 0.00057711 & 0.00062766 & 0.00052184 & 0.00053499 \\
\hline $\begin{array}{l}\text { PSNR( peak } \\
\text { signal to } \\
\text { noise ratio) }\end{array}$ & $32.3874 \mathrm{~dB}$ & $32.0227 \mathrm{~dB}$ & $32.8246 \mathrm{~dB}$ & $32.7165 \mathrm{~dB}$ \\
\hline $\begin{array}{l}\text { S/MSE(signal } \\
\text { to mean } \\
\text { square error) }\end{array}$ & $24.7756 \mathrm{~dB}$ & $24.4109 \mathrm{~dB}$ & $25.2128 \mathrm{~dB}$ & $25.1047 \mathrm{~dB}$ \\
\hline $\begin{array}{l}\text { SNR(signal } \\
\text { to noise ratio) }\end{array}$ & $19.3170 \mathrm{~dB}$ & $18.8474 \mathrm{~dB}$ & $19.6605 \mathrm{~dB}$ & $19.5071 \mathrm{~dB}$ \\
\hline
\end{tabular}

It is clear from table 1 that de-noised image using wave atom with new threshold has lowest mean square error (MSE), highest peak signal to noise ratio (PSNR), highest signal to mean square error (S/MSE) and highest signal to noise ratio (SNR). It is also clear from the table that quality parameters of image de-noised by wave atom is better as comparison to the quality parameters of image de-noised by wavelet. 

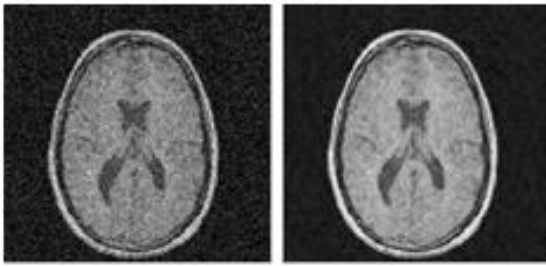

(c)PSNR= 24.6166

(d)PSNR $=26.107$

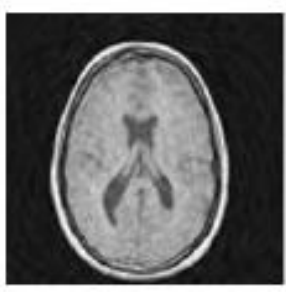

(e)PSNR $=25.8739$

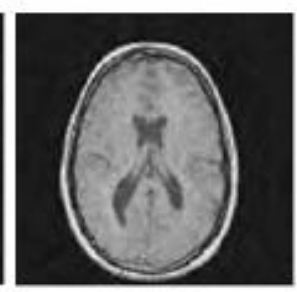

Fig 2:Low SNR Images

(a)Noisy image

(b) De-noised using Wave Atom with old threshold

(c) De-noised using Wavelet with old threshold

(d) De-noised using Wave Atom with new threshold

(e) De-noised using Wavelet with new threshold

Different quality parameters for above denoised images are given in table 2 .

Table 2. Quality Parameters for Low SNR Images

\begin{tabular}{|l|l|l|l|l|}
\hline $\begin{array}{l}\text { Quality } \\
\text { parameters }\end{array}$ & $\begin{array}{l}\text { Denoised } \\
\text { using } \\
\text { Wave } \\
\text { Atom } \\
\text { with old } \\
\text { threshold }\end{array}$ & $\begin{array}{l}\text { De- } \\
\text { noised } \\
\text { using } \\
\text { Wavelet } \\
\text { with old } \\
\text { threshold }\end{array}$ & $\begin{array}{l}\text { De- } \\
\text { noised } \\
\text { using } \\
\text { Wave } \\
\text { Atom } \\
\text { with new } \\
\text { threshold }\end{array}$ & $\begin{array}{l}\text { De- } \\
\text { noised } \\
\text { using } \\
\text { Wavelet } \\
\text { with new } \\
\text { threshold }\end{array}$ \\
\hline $\begin{array}{l}\text { MSE(mean } \\
\text { square error) }\end{array}$ & 0.0031931 & 0.0034542 & 0.0024507 & 0.0025859 \\
\hline $\begin{array}{l}\text { PSNR( peak } \\
\text { signal to } \\
\text { noise ratio) }\end{array}$ & $24.9578 \mathrm{~dB}$ & $24.6166 \mathrm{~dB}$ & $26.107 \mathrm{~dB}$ & $25.8739 \mathrm{~dB}$ \\
\hline $\begin{array}{l}\text { S/MSE(signal } \\
\text { to mean } \\
\text { square error) }\end{array}$ & $17.346 \mathrm{~dB}$ & $17.0047 \mathrm{~dB}$ & $18.4952 \mathrm{~dB}$ & $18.2621 \mathrm{~dB}$ \\
\hline $\begin{array}{l}\text { SNR(signal } \\
\text { to noise ratio) }\end{array}$ & $13.3389 \mathrm{~dB}$ & $12.7882 \mathrm{~dB}$ & $15.3178 \mathrm{~dB}$ & $14.7623 \mathrm{~dB}$ \\
\hline
\end{tabular}

\section{CONCLUSIONS}

A better scheme is presented for the denoising of magnetic resonance imaging using wave atom transform. It is proved that the proposed threshold provides a better quality on MRI as compared to old threshold. The edge preserving property is clearly an advantage of the proposed method.

\section{REFERENCES}

[1] L. Demanet, L.Ying, "Wave atoms and sparsity of oscillatory patterns," Appl Comput Harmon Anal., vol. 23, no. 3, pp.368-387, 2007.

[2] G.A.Wright, "Magnetic Resonance Imaging,” IEEE Signal Processing Magazine, vol. 14, no. 1, pp. 56-66, January 1997.

[3] J. Sijbers, D. H. J. Poot, A. J. den Dekker and W. Pintjens, "Automatic estimation of the noise variance from the histogram of a magnetic resonance image," Physics in Medicine and Biology, vol. 52, no. 5, pp. 1335-1348, February 2007.

[4] J. B. Weaver, Y. Xu, D. M. Healy Jr., and L. D. Cromwell. Filtering noise from images with wavelet transforms. Magn Reson Med 1991; 21:2:288-295.

[5] R. D. Nowak. Wavelet-based Rician noise removal for magnetic resonance imaging. IEEE Trans Image Process 1999; 8:10:1408-1419.

[6] M. Wink and J. B. T. M. Roerdink. Denoising functional MR images: A comparison of wavelet denoising and Gaussian smoothing. IEEE Trans Image Process 2004; 23:3:374-387.

[7] A. Pizurica, A. M. Wink, E. Vansteenkiste, W. Philips, and J. B. T. M. Roerdink. A review of wavelet denoising in MRI and ultrasound brain imaging. Current Med Imag Rev 2006; 2:2:247-260.

[8] D. Tisdall and M. S. Atkins. MRI denoising via phase error estimation. Proc SPIE Med Imag 2005; 646-654.

[9] Gerlind Plonka and Jianwei Ma. Nonlinear Regularised Reaction-Diffusion Filter for Denoising of Images with Textures. IEEE Trans. Image Processing 2008; $17: 8: 1283-1294$.

[10] J. Rajeesh, R .S. Moni, S. Palani Kumar, T. Gopalakrishnan,"Noise Reduction in Magnetic Resonance Images using Wave Atom Shrinkage," International Journal of Image Processing, vol. 4, issue 2, pp. 131-141, March/April 2010.

[11] J. Rajeesh, R. S. Moni, S. Palani Kumar, T. Gopalakrishnan, "Rician Noise Removal on MRI Using Wave Atom Transform with Histogram Based Noise Variance Estimation," IEEE Communication Control and Computing Technologies, pp.531-535, December 2010.

[12] P. Gravel, G. Beaudoin, J. A. De Guise, "A method for modeling noise in medical images," IEEE Trans. Med. Imag., vol. 23, no. 10, pp. 1221- 1232, October 2004

[13] C. G. Koay and P. J. Basser, "Analytically exact correction scheme for signal extraction from noisy magnitude MR signals," J. Magn. Reson. Vol. 179, no. 2, pp.317-322, April 2006.

[14] G. Cottet, L. Germain. Image processing through reaction combined with nonlinear diffusion. Math Comput 1993; vol 61:pp.659-673.

[15] [Online] .Available: http://www.mathworks.com/Matlab central/fx_files/14237/3/content/rician/html/ricedemo.ht $\mathrm{ml}$.

[16] [Online]. Available: http://www.oasis-brain.org/ 